\title{
Risk factors and mortality in the Carbapenem-resistant Klebsiella pneumoniae infection: case control study
}

\author{
Fethiye Akgul, Ilkay Bozkurt, Mustafa Sunbul, Saban Esen, \\ Hakan Leblebicioglu
}

Department of Clinical Microbiology and Infectious Diseases, School of Medicine, Ondokuz Mayis University, Samsun, Turkey

Carbapenem-resistant Klebsiella pneumoniae (CRKP) has been known as a nosocomial pathogen, both for the last 10 years in Turkey and for 20 years worldwide. Due to limited treatment options and high mortality rates, despite improvements in the field of medicine at the present time, CRKP is still a big threat for public health. This study was carried out between the dates of January 2010 and September 2014. Patients $\geq 18$ who were hospitalized for at least $72 \mathrm{~h}$ and who also had CRKP growth were included in the study as a case group. In the same period patients, who were hospitalized in the same ward and did not have CRKP growth were selected as the control group. It was determined that no glycopeptides and steroids use nor tracheostomy as protective factors would be employed in terms of non-development of CRKP. Mechanical ventilation, tracheostomy, urinary catheter presence, central venous catheterization, nasogastric tube placement, advanced age, acute renal insufficiency, total parenteral nutrition, carbapenem, glycopeptide, and piperacillin tazobactam were all detected as risk factors in terms of CRKP infection development. As a result, rational usage of antibiotics for preventing infections developing with CRKP should be targeted.

Keywords: Carbapenem-resistant klebsiella pneumoniae, klebsiella pneumonia, Risk factors, Mortality

\section{Introduction}

Infections developing with resistant micro-organisms have become a major problem threatening community health care, especially in recent years. ${ }^{1}$ Faced with the extended spectrum beta-lactamase (ESBL) problem in the 1990s, carbapenemases, producing Enterobacteriaceae became the current issue in the later 1990s and early 2000s. CRKP isolates were identified for the first time in North Carolina in $1996 .{ }^{2}$ Identification was started in our country in the years of $2000 .{ }^{3}$ CRKP has a great role in the common appearance of carbapenem-resistant Enterobacteriaceae. ${ }^{4}$ In the infections effects of the disease, CRKP progresses directly, and increases the length of hospital stay, cost, and mortality. ${ }^{5,6}$ Mortality may be decreased in such kind of infections with the early determination of risk factors. If we detect high-risk patients early, we can administer empiric antibiotic treatment which covers possible resistant pathogens. Besides this, we can prevent the spread of micro-organisms through the hospital by rational use of antibiotics and taking infection control measures. ${ }^{7,8}$

Correspondence to: Ilkay Bozkurt, Department of Clinical Microbiology and Infectious Diseases, School of Medicine, Ondokuz Mayis University. Email: drilkaybozkurt@gmail.com
We aimed to determine risk factors and mortality in patients infected with CRKP by comparing with a control group.

\section{Materials and methods}

This study was carried out between January 2010 and September 2014. Patients, who were hospitalized at least 72 $h$ in the hospital, were $\geq 18$ years, and had CRKP growth in microbiological culture were included in the study. All the isolates were accepted as a causative agent by an infectious diseases specialist, and antibiotic therapy against CRKP was given. The study was organized as a case-control study. A control group was selected among patients who were hospitalized at least $72 \mathrm{~h}$ in the same wards and period with the cases. The control group had no CRKP growth from clinical samples. For each case, at least one control patient was determined: 95 cases and 100 controls were included in the study. The only inclusion criteria for control group members were being in the same ward in the same period as the CRKP patients. Minimum inhibitory concentrations of antibiotics were determined by $E$-test. CLSI breakpoints were used. We did not perform routine surveillance sampling, either in case or control groups.

The medical records of patients were obtained from the database of the hospital. The demographic 


\section{Number of Case}

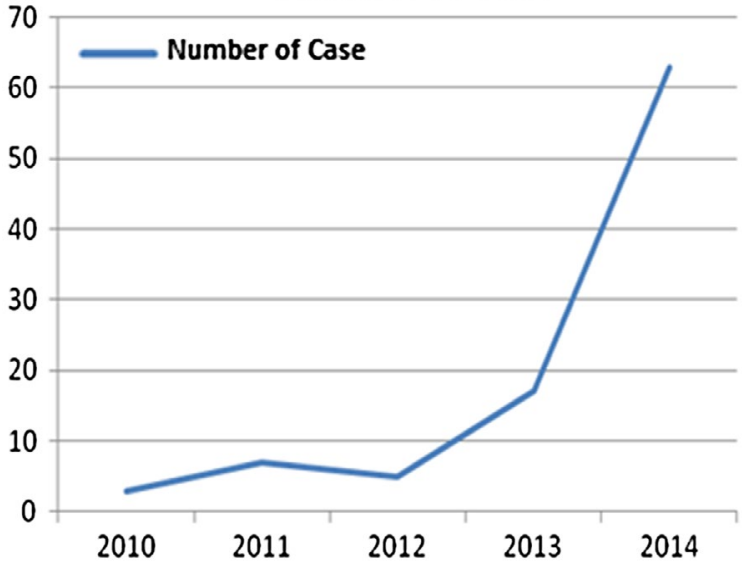

Graph 1 Distribution of cases according to the years

characteristics of the patients (age, gender), symptoms and clinical findings, comorbid conditions, performed invasive procedures, central venous catheter (CVC), urinary catheterization, nasogastric $(\mathrm{NG})$ catheter, tracheostomy and endotracheal intubation, emergency or elective surgery history, special treatments (corticosteroids, chemotherapy, blood products), staying in the intensive care unit (ICU), staying in the hospital within the last three months, history of transferring from another hospital, positive sample source, and antibiotic therapy use by the patients were assessed. Peripheral blood, catheter, cerebrospinal fluid, sputum, tracheal aspirates, urine, and pleural fluid cultures were taken in accordance with the localization of the infection.

The presence of another bacterial growth simultaneously with CRKP, length of hospital as well as ICU stay, duration of stay in mechanical ventilator (MV), and medical prognosis of the patients were assessed retrospectively. As the control group was selected randomly, it included patients not having growth in their culture as well.

Data analysis was conducted in the SPSS Statistics 21.0 package program for Windows. Results were submitted in the form of frequency, percentage, median (minimummaximum), and arithmetic mean \pm standard deviation. For the categorical variables, the Pearson chi-square continuity correction and Fisher's exact test analyses were used. Normality tests of non-categorical variables were performed with the Kolmogorow-Smirnov test. For group comparisons, two sample $t$-tests independent from parametric methods and the Mann-Whitney $U$ test from non-parametric methods were used. Factors having effect on prognosis were investigated by binary logistic regression analysis.

As a result of univariate statistical analyses, variables showing significant differences among case and control groups were included in a multivariate logistic regression model, where values of. $p<0.05$ were considered statistically significant. The local ethical approval was obtained from the Ondokuz Mayis University School of Medicine.

\section{Results}

In the case group, 63 of 93 patients $(66.3 \%)$ were male, as compared to 62 of 100 patients $(62 \%)$ in the control group. The mean age of case and control group patients was 66 (19-94 years) and 58 (21-87 years), respectively. The groups were similar in terms of age and gender $(p<0.05)$. The number of cases increased each year between the years 2010-2014.

Positive blood culture results received from the catheter were found to be more frequent for the case group than the control group $(p<0.05)$ (Table 1$)$. In terms of comorbid conditions, no significant difference was found among the groups, other than acute renal insufficiency (ARI) $(p<0.05)$. Diarrhea was much more frequent in patients having CRKP growth $(p<0.05)$. Interventions made within the period of hospitalization of the patients are given in Table 2.

It was detected that a history of hospitalization was much more frequent in patients having CRKP growth when staying of patients in a nursing home, transferring from another hospital, hospitalization history in hospital, and need of ICU was compared $(p<0.05)$. In the case group, 80 patients $(84.2 \%)$ had history of urinary catheter placement $(p<0.05)$ (Table 2$)$. The number of days for patients with mechanical ventilation (when patients depended on mechanical ventilation) was an average of $22.9 \pm 19.9$ days in the case group, and $7.6 \pm 7.6$ days in the control group $(p=0.001)$. While the length of hospital stay was 31 days (4-160 days) until growth was detected in the case group, the total length of hospitalization was 16 days ( $4-150$ days) in the control group $(p<0.001)$. The length of stay in ICU was $25.7 \pm 23.6$ in the case group, and $13.3 \pm 11.8$ days in the control group $(p<0.05)$.

In the case group, 87 patients $(91.6 \%)$ had a history of antibiotic use, as compared to 82 patients $(82 \%)$ in the control group $(p=0.079)$. Carbapenem $(47 / 28$, $p<0.05)$, glycopeptide $(40 / 9, p<0.001)$, colistin $(15 / 5$, $p<0.05)$, and piperacillin tazobactam $(39 / 21, p<0.05)$ use was much more common in the case group in comparison to the control group. Other antibiotics used were fluoroquinolones, daptomycin, aminoglycosides, cephalosporins, ampicillin + sulbactam, linezolid, trimethoprim + sulfamethoxazole (TMP-SMX), metronidazole,

Table 1 Distribution of the positive culture results according to clinical samples

\begin{tabular}{lcc}
\hline & Case & Control \\
\cline { 2 - 3 } Sampling & $n: \mathbf{9 5}(\%)$ & $n: \mathbf{1 0 0}(\%)$ \\
\hline Blood culture & $32(33.7)$ & $24(24)$ \\
Blood culture from catheter & $11(11.6)$ & $3(3)$ \\
Cerebrospinal fluid culture & 0 & $1(1)$ \\
Tracheal aspirate culture & $13(13.7)$ & $6(6)$ \\
Urine culture & $8(8.4)$ & $14(14)$ \\
Urine culture (catheter) & $27(28.4)$ & $25(25)$ \\
Exudate culture & $13(13.7)$ & $11(11)$ \\
Sputum culture & $2(2.1)$ & $1(1)$ \\
\hline
\end{tabular}


Table 2 Interventions applied to the patients

\begin{tabular}{lccc}
\hline & Case group & Control group & \\
\cline { 2 - 3 } Variables & $\boldsymbol{N}=\mathbf{9 5}(\%)$ & $\boldsymbol{N}=\mathbf{1 0 0 ( \% )}$ & $\boldsymbol{P}$ value \\
\hline Mechanical ventilation & $39(41.1)$ & $23(23)$ & $<0.05$ \\
Tracheostomy & $18(18.9)$ & $3(3)$ & $<0.001$ \\
Colostomy & $6(6.3)$ & $7(7)$ & 1.0 \\
Urinary catheter & $80(84.2)$ & $65(65)$ & $<0.05$ \\
presence & & & \\
Cystostomy & $1(1.1)$ & $2(2)$ & 1.0 \\
Nephrostomy & $5(5.3)$ & $4(4)$ & 0.74 \\
Nasogastric tube & $52(54.7)$ & $23(23)$ & $<0.001$ \\
presence & & & \\
Central venous & $62(65.3)$ & $33(33)$ & $<0.001$ \\
catheter & & & \\
History of operations & $38(40)$ & $33(33)$ & 0.3 \\
History of emergency & $13(35.1)$ & $4(12.1)$ & $<0.05$ \\
operations & & & \\
Chest drain & $8(8.4)$ & $2(2)$ & 0.054 \\
Drain & $24(25.3)$ & $18(18)$ & 0.29 \\
Gastrostomy & $6(6.3)$ & $2(2)$ & 0.16 \\
Bronchoscopy & $2(2.1)$ & $1(1)$ & 0.61 \\
Endoscopy/colonos- & $12(12.6)$ & $10(10)$ & 0.72 \\
copy & & & \\
Hemodialysis & $10(10.5)$ & $9(9)$ & 0.90 \\
Bedside debridement & $15(15.8)$ & $16(16)$ & 1.0 \\
Ventricular drainage & $4(4.2)$ & 0 & 0.055 \\
Bone marrow biopsy & $15(15.8)$ & $11(11)$ & 0.44 \\
ERCP & $5(5.3)$ & $3(3)$ & 0.48 \\
TPN & $29(30.5)$ & $14(14)$ & $<0.05$ \\
\hline
\end{tabular}

ERCP: Endoscopic retrograde cholangiopancreatography, TPN: Total parenteral nutrition, NG: Nasogastric Tube.

fosfomycin, tigecycline, rifampicin, and clarithromycin. In 27 of 95 patients (28.4\%) involved in the case group, another bacterial infection was detected simultaneously with CRKP. Eleven out of 95 cases were discharged with recovery, 16 patients died. We found no relationship between mortality and the presence of simultaneous bacterial infection in the case group $(p>0.05)$.

As a result of univariate statistical analyses, $p<0.05$ variables determined as risk factors for CRKP development were: ARI, steroids, diarrhea, need of mechanical ventilation support, tracheostomy presence, urinary catheter, CVC or invasive device use, NG, emergency operation, use of blood products, total parenteral nutrition (TPN), hospitalization history in the hospital, carbapenem, glycopeptide, colistin and piperacillin tazobactam use, and a multiple univariate logistic regression model. It was found that non-use of glycopeptide (Odds ratio: 0.143; \%95 Confidence interval: 0.031-0.674; $p<0.05$ ), non-use of steroids (Odds ratio: 0.244; \%95 Confidence interval: $0.072-0.822 ; p<0.05)$ and the absence of tracheostomy (Odds ratio: 0.06; \%95 Confidence interval: 0.006-0.614; $p<0.05)$ were protective factors in terms of non-development of CRKP infection. A total of $47.4 \%$ of the patients in the case group and $18 \%$ of the patients in the control group died $(p<0.001)$.

\section{Discussion}

CRKP has an important place among nosocomial agents, particularly in recent years. CRKP causes a nosocomial infection because it lives and spreads easily around the hospital environment, and acquires resistance against antibiotics. ${ }^{9} 10$

As the carbapenem resistance in the Enterobacteriaceae family was elevating from 1 to $4 \%$, CRKP rate elevated from 2 to $10 \%$ between the years 2001 and 2011. In this study, the CRKP rate has increased significantly in recent years and has reached $66.9 \%$ in 2014 , despite the fact that it was only $3.2 \%$ in 2010.

In this study, factors such as hospital-stay history, mechanical ventilation, tracheostomy, urinary catheter presence, $\mathrm{CVC}, \mathrm{NG}$ placement, advanced age, acute renal failure, TPN, and use of antibiotics (carbapenem, glycopeptide, piperacillin tazobactam) were all determined as risk factors for CRKP development. On the other hand, non-use of glycopeptide and steroids, and the absence of tracheostomy prevented the development of CRKP. Similar factors have been defined as risk factors for CRKP infection/colonization in the literature ${ }^{11-14}$.

In the previous studies, most of the control groups had been selected from patients who were infected with carbapenem-sensitive K. pneumoniae isolates. However, this may cause bias. The risk factors may reflect the protective factors for the carbapenem-susceptible isolates rather than real risk factors for the CRKP. ${ }^{15-18}$ In order to eliminate the bias, our control group was randomly selected from patients with no CRKP growth.

Mortality rate was between $24-65 \%$ in patients infected with CRKP. Carbapenem resistance has been defined as an independent risk factor for mortality. ${ }^{19-21}$ The mortality rate $(47.4 \%)$ was found to be significantly higher in patients with CRKP growth $(p<0.001)$. Mortality was higher in the case group due to underlying diseases of the patients, the limited treatment options, long length of stay in hospital, a growing number of procedures, and how closely these conditions were related to each other. The CRKP-associated mortality rate was compatible with previous studies, which were also over $40 \% .^{22,23}$ In this study, non-use of steroids was detected as a protective factor in terms of CRKP development (Odds ratio: 0.244; \%95; CI: $0.072-0.822 ; p<0.05)$. However, in contrast to our study, Bremmer et al. ${ }^{24}$ demonstrated that systemic steroids use is not a risk factor for CRKP development. It has been thought that different results may arise from the selection of the patient population. The commonly used antibiotics for nosocomial infections, such as antipseudomonal penicillins, carbapenems, glycopeptides, and colistin were determined as risk factors for the development of CRKP infection. ${ }^{12,25-30}$ However Kofteridis et al..$^{31}$ did not find any relationship between CRKP and antimicrobial treatment. The use of carbapenem $(p<0.05)$, glycopeptide $(p<0.001)$, piperacillin tazobactam $(p<0.05)$ and colistin $(p<0.05)$ was determined as the risk factor in patients.

Due to the huge number of patients, smaller healthcare staff, invasive procedures, frequent wide-spectrum antimicrobial use, and horizontal transmission of clonal strains are all critical factors for the spread of antibiotic 
resistance..$^{32,33}$ The length of hospital (particularly ICU) stay is closely related to CRKP colonization and infection risk increases as the patient stays longer in the ICU. As a matter of fact, $75 \%$ of the patients have been determined to be colonized with Klebsiella pneumoniae Carbapenemase (KPC) during the ICU stay. ${ }^{34}$ There was no statistically significant difference for the need care in the ICU between the two groups. The ICU stay was not identified as a risk factor in terms of CRKP infection because we selected similar numbers of case and control groups from the medical and surgical units and also ICUs. Patients in ICUs stay longer, use mechanical ventilation, the longer duration of exposure to risk factors such as $\mathrm{NG}, \mathrm{CVC}$, catheter, and potential risk for widely spectrum antibiotic use due to the increased length of ICU. The length of hospital stay $(p<0.001)$, ICU stay $(p<0.05)$ and prolonged duration of mechanical ventilation stay have been found as risk factors in terms of the development of CRKP $(p<0.001)$.

Longer length of hospital and also ICU stay, mechanical ventilation, the presence of tracheostomy, emergency surgical intervention, presence of urinary catheter, and NG placement are basic risk factors determined in this study for the development of CRKP infection, and indications of invasive procedures should be assessed in the light of those specified. If NG or urinary catheter is nonessential, it should be removed. As soon as the patient's need for ICU ends, active surveillance should be performed. Preemptive contact isolation precautions should be taken in patients with high colonization/infection risk of CRKP. According to hospital conditions, for patients with positive CRKP, cohort region should be constituted and cohort for patient and staff should be performed. Staff training is one of the most important measures for prevention and control of infections that are connected to each other 'like dominoes.' For this reason, the staff should be informed, and its interests should be kept alive and supervised by recurrent trainings.

Consequently, hospital-based policies should be developed in order to minimize infections caused by CRKP. Use of unnecessary antibiotics, notably carbapenems and glycopeptides should be avoided as in this study.

\section{Disclosure statement}

None to declare.

\section{ORCID}

Hakan Leblebicioglu (D) http://orcid.org/0000-0002-60338543

\section{References}

1 Tängdén T, Giske CG. Global dissemination of extensively drugresistant carbapenemase-producing Enterobacteriaceae: clinical perspectives on detection, treatment and infection control. J Intern Med. 2015;277:501-512.

2 Doi Y, Paterson DL. Carbapenemase-producing Enterobacteriaceae. Semin Respir Crit Care Med. 2015;36:74-84.
3 Gülmez D, Woodford N, Palepou MF, et al. Carbapenem-resistant Escherichia coli and Klebsiella pneumoniae isolates from Turkey with OXA-48-like carbapenemases and outer membrane protein loss. Int J Antimicrob Agents. 2008;31:523-526.

4 Guh AY, Bulens SN, Mu Y, et al. Epidemiology of carbapenemresistant Enterobacteriaceae in 7 US communities, 2012-2013. JAMA. 2015;314:1479-1487.

5 Löfmark S, Sjöström K, Mäkitalo B, et al. Carbapenemase-producing Enterobacteriaceae in Sweden 2007-2013: experiences from seven years of systematic surveillance and mandatory reporting. Drug Resist Updat. 2015;20:29-38.

6 Viale P, Giannella M, Lewis R, et al. Predictors of mortality in multidrug-resistant Klebsiella pneumoniae bloodstream infections. Expert Rev Anti Infect Ther. 2013;11:1053-1063.

7 Carvalhaes CG, Cayô R, Gales AC. Klebsiella pneumoniae carbapenemase-producing Klebsiella pneumoniae in the intensive care unit: a real challenge to physicians, scientific community, and society. Shock. 2013;39(Suppl. 1):32-37.

8 Yamamoto M, Pop-Vicas AE. Treatment for infections with carbapenem-resistant Enterobacteriaceae: what options do we still have? Crit Care. 2014;18:229.

9 Berezin BE, Towner KJ. Acinetobacter spp as nosocomial pathogens. microbiological, clinical and epidemiological features. Clin Microbiol Rev. 1996;9:148-165.

10 Nordmann P. Carbapenemase-producing Enterobacteriaceae: overview of a major public health challenge. Med Mal Infect. 2014; $44: 51-56$.

11 Candevir Ulu A, Kurtaran B, Inal AS, et al. Risk factors of carbapenemresistant Klebsiella pneumoniae infection: a serious threat in ICUs. Med Sci Monit. 2015;21:219-224.

12 Cardoso T, Ribeiro O, Aragão IC, et al. Additional risk factors for infection by multidrug-resistant pathogens in healthcare-associated infection: a large cohort study. BMC Infect Dis. 2012;12:375.

13 Jiao Y, Qin Y, Liu J, et al. Risk factors for carbapenem-resistant Klebsiella pneumoniae infection/colonization and predictors of mortality: a retrospective study. Pathog Glob Health. 2015;109:6874.

14 Kofteridis DP, Valachis A, Dimopoulou D, et al. Risk factors for carbapenem-resistant Klebsiella pneumoniae infection/colonization: a case-case-control study. J Infect Chemother. 2014;20:293-297.

15 Falagas ME, Rafailidis PI, Kofteridis D, et al. Risk factors of carbapenem-resistant Klebsiella pneumoniae infections: a matched case control study. J Antimicrob Chemother. 2007;60:1124-1130.

16 Gasink LB, Edelstein PH, Lautenbach E, et al. Risk factors and clinical impact of Klebsiella pneumoniae carbapenemase-producing K. pneumoniae. Infect Control Hosp Epidemiol. 2009;30:1180-1185.

17 Hussein K, Sprecher H, Mashiach T, Oren I, Kassis I, Finkelstein R. Carbapenem resistance among Klebsiella pneumoniae isolates: risk factors, molecular characteristics, and susceptibility patterns. Infect Control Hosp Epidemiol. 2009;30:666-671.

18 Patel G, Huprikar S, Factor SH, et al. Outcomes of carbapenemresistant Klebsiella pneumoniae infection and the impact of antimicrobial and adjunctive therapies. Infect Control Hosp Epidemiol. 2008;29:1099-1106.

19 Daikos GL, Markogiannakis A. Carbapenemase-producing Klebsiella pneumoniae: (when) might we still consider treating with carbapenems? Clin Microbiol Infect. 2011;17:1135-1141.

20 Deshpande LM, Rhomberg PR, Sader HS, et al. Emergence of serine carbapenemases (KPC and SME) among clinical strains of Enterobacteriaceae isolated in the United States medical centers: report from the MYSTIC program (1999-2005). Diagn Microbiol Infect Dis. 2006;56:367-372.

21 Schwaber MJ, Klarfeld-Lidji S, Navon-Venezia S, et al. Predictors of carbapenem-resistant Klebsiella pneumoniae acquisition among hospitalized adults and effect of acquisition on mortality. Antimicrob Agents Chemother. 2008;52:1028-1033.

22 Tumbarello M, Trecarichi EM, De Rosa FG, et al. ISGRI-SITA (Italian Study Group on Resistant Infections of the Società Italiana Terapia Antinfettiva). infections caused by KPC-producing Klebsiella pneumoniae: differences in therapy and mortality in a multicentre study. J Antimicrob Chemother. 2015;70:2133-2143.

23 Hoxha A, Kärki T, Giambi C et al. Attributable mortality of carbapenemresistant Klebsiella pneumoniae infections in a prospective matched cohort study in Italy, 2012-2013. J Hosp Infect. doi: 10.1016/j.jhin.2015.06.018. [Epub ahead of print] PubMed PMID: 26319590 .

24 Bremmer DN, Clancy CJ, Press EG, et al. KPC-producing Klebsiella pneumoniae strains that harbor $\mathrm{AAC}\left(6^{\prime}\right)$-Ib exhibit intermediate resistance to amikacin. Antimicrob Agents Chemother. 2014;58:75977600 . 
25 Falagas ME, Rafailidis PI, Kofteridis D, et al. Risk factors of carbapenem-resistant Klebsiella pneumoniae infections: a matched case control study. J Antimicrob Chemother. 2007;60:1124-1130.

26 Hyle EP, Ferraro MJ, Silver M, Lee H, Hooper DC. Ertapenemresistant Enterobacteriaceae risk factors for acquisition and outcomes. Infect Control Hosp Epidemiol. 2010;31:1242-1249.

27 Hussein K, Sprecher H, Mashiach T, et al. Carbapenem resistance among Klebsiella pneumoniae isolates: risk factors, molecular characteristics, and susceptibility patterns. Infect Control Hosp Epidemiol. 2009;30:666-671.

28 Jeon MH, Choi SH, Kwak YG, Chung JW, Lee SO, Jeong JY, et al. Risk factors for the acquisition of carbapenem-resistant Escherichia coli among hospitalized patients. Diagn Microbiol Infect Dis. 2008;62:402-406.

$29 \mathrm{Wu}$ D, Cai J, Liu J. Risk factors for the acquisition of nosocomia infectio with carbapenem-resistant Klebsiella pneumoniae. South Med J. 2011;104:106-110.
30 Dizbay M, Guzel Tunccan O et al. Emergence of carbapenemresistant Klebsiella spp. infections in a Turkish University hospital: epidemiology and risk factors. J Infect Dev Ctries. 2014;8:44-49.

31 Kofteridis DP, Valachis A, Dimopoulou D, et al. Risk factors for carbapenem-resistant Klebsiella pneumoniae infection/colonization: a case-case-control study. J Infect Chemother. 2014;20:293-297.

32 Hyle EP, Lipworth AD, Zaoutis TE, et al. Risk factors for increasing multidrug resistance among extended-spectrum beta-lactamaseproducing Escherichia coli and Klebsiella species. Clin Infect Dis. 2005;40:1317-1324.

33 Brusselaers N, Vogelaers D, Blot S. The rising problem of antimicrobial resistance in the intensive care unit. Ann Intensive Care. 2011;1:47.

34 Papadimitriou-Olivgeris M, Marangos M, Fligou F, et al. KPCproducing Klebsiella pneumoniae enteric colonization acquired during intensive care unit stay: the significance of risk factors for its development and its impact on mortality. Diagn Microbiol Infect Dis. 2013;77:169-173. 